\title{
Wie verhalte ich mich im Gefahrenfall in der Zahnarztpraxis?
}

\author{
Auch in der Zahnarztpraxis kann das Thema Selbstverteidigung bei einem Angriff lebensrettend \\ sein. Beim Treffen der Bezirksgruppe Merzig-Saarlouis ging es deshalb Anfang März um das rich- \\ tige Verhalten in einem solchen Ausnahmefall.
}

Referent Martin Hoffmann, stellvertretender Leiter des Polizeibezirks Saarlouis und Leiter der Dragon-Wing-Tsun-Academy in Dillingen (Saar) gab zum Thema „Aktuelle Sicherheitsaspekte in der Zahnarztpraxis - Anwendungen und praktische Handlungsmöglichkeiten im Gefahrenfall“ Tipps und Tricks, wie man richtig deeskaliert und wie man sich am besten in Notsituationen verhält. Hoffmann hat des Weiteren einen Alarmplan für die Zahnarztpraxis erstellt, der für jedes Praxisteam einfach umzusetzen ist. Bei der anschließenden Gesprächsrunde wurde angeregt über das richtige Verhalten im Notdienst diskutiert. Auch hier konnte Hoffmann mit Witz und Charme gute Hilfestellungen geben. Im Mai und Juni soll es vier Selbstverteidi-

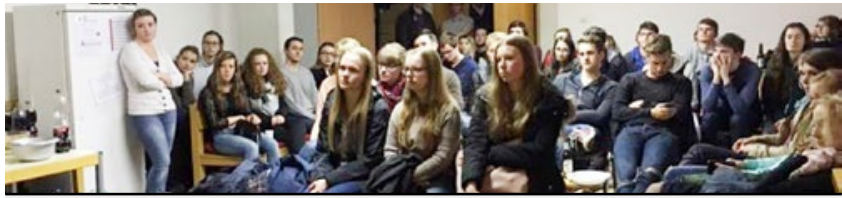

Informationsabend an der Universität des Saarlandes

Von Studenten für Studenten

Unter dem Motto "Ein Staffellauf der besonderen Art" haben über 60 Studierende an einem Informationsabend an der Universität Homburg teilgenommen. Ziel des Studentenabends im Partykeller des Studentenwohnheims A war es, die Erfahrungen der höheren Semester an die niedrigeren Semester weiterzugeben. Wie bestehe ich die Hürden des Studiums? Wie meistere ich Klausuren, mündliche Prüfungen und die praktischen Kurse? Dies waren nur einige Fragen, die den jüngeren Studierenden unter den Nägeln brannten. Weiterhin gab es wertvolle Literaturtipps und Hinweise zu Lernstrategien. Amüsante Anekdoten der Studierenden, sowie die kulinarische Versorgung durch den FVDZ-Landesverband Saarland sorgten für ausgelassene Stimmung unter den Teilnehmern.

Dirk Ruffing, Mitglied im Bundesvorstand des Freien Verbandes Deutscher Zahnärzte (FVDZ), berichtete von seiner Famulatur auf Samoa und beantwortete Fragen zum Freien Verband. Die Studentenbeauftragte des Verbandes, Birgit Hauer, reiste eigens aus Bonn an und informierte über die Planung einer Auslandsfamulatur. Weiterhin berichtete ein junger Assistenzzahnarzt von seiner Vorbereitung auf das Examen, die Examenszeit und die spätere Arbeitssuche.

Die positive Resonanz der Studenten war deutlich spürbar. Der Informationsabend war die best besuchteste Veranstaltung seit Einführung der Studentenarbeit im Saarland. Auch die anwesenden Mitglieder des saarländischen Landesvorstandes des FVDZ, Dr. Natascha Bauer und Dr. Dr. Mike Jacob verspürten den deutlichen Wunsch der Studierenden, diese Veranstaltungen zur Tradition werden zu lassen.

red gungskurse in der Wing-Tsun-Academy unter der Leitung von Hoffmann geben, die auf diesem Thema weiter aufbauen und den Teilnehmern nicht nur verbal Schlagkraft verleihen sollen. Mit 18 Personen aus sieben Praxisteams war das Interesse an diesem Thema sehr groß.

\section{ZoRA-Stammtisch und Bezirksgruppentreffen}

Bereits im Februar traf sich in Saarbrücken im Traditionsgasthaus Undine die Bezirksgruppe Süd unter dem Vorsitz von Dr. Natascha Bauer in Kombination mit dem bestehenden ZoRA Stammtisch unter der Leitung von Dr. Christiane Wagner. Schon an der höheren Männerquote im Saal der Undine in Saarbrücken war die Neuerung unschwer zu erkennen.

Zunächst referierte Martin Hoffmann zu dem Thema „Wie wehre ich mich in Notsituationen?". Wie schon oben geschildert, gestaltete Hoffmann seinen Vortrag so kurzweilig, dass sich alle Teilnehmer spontan zu seinem Selbstverteidigungskurse in der Wing-Tsun-Academy anmelden wollten.

Der zweite Teil des Abends wurde gestaltet von Sanitätsrat Dr. Hans-Joachim Lellig, Vorsitzender der Ärztekammer des Saarlandes -Abt. Zahnärzte - zum Thema „GÖ̈. Was kommt auf uns zu? Wie können wir Schlimmeres abwenden?" Er drückte sein Unverständnis über die Akzeptanz der geplanten GOÄ-Reform in der Ärzteschaft aus. Auch ließ er in seinen Ausführungen deutlich erkennen, dass die Zahnärzteschaft kaum Einfluss auf die Entscheidungen der Ärzte nehmen kann. Ein ernüchternder Beitrag, den Lellig humorvoll und positiv in die Zukunft schauend vortrug.

Jenseits des fachlichen war ihm ein besonderes Anliegen, für die von ihm im Saarland initiierten Veranstaltungen von „special olymipcs“ zu werben. Die Auftaktveranstaltung 2015 war auch dank der Mithilfe von Studenten und Kolleginnen des FVDZ ein voller Erfolg.

Selbstverständlich kam auch dieses Mal nach dem informativen Fortbildungsteil das gemütliche Beisammensein nicht zu kurz. Beim gemeinsamen Essen gab es einen regen Gedankenund Ideenaustausch unter den anwesenden Damen und Herren.

Dr. Daniela Wind

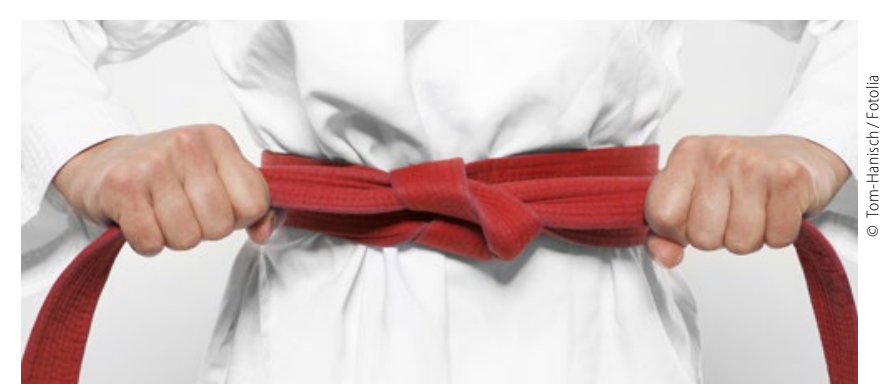

